\title{
Micro-Cavity Effect of ZnO/Ag/ZnO Multilayers on Green Quantum Dot Light-Emitting Diodes
}

\author{
Hyungin Lee and Jiwan Kim ${ }^{\dagger}$ \\ Department of Advanced Materials Engineering, Kyonggi University, Suwon 16227, Korea \\ (Received February 5, 2018; Revised March 8, 2018; Accepted March 9, 2018)
}

\begin{abstract}
$\mathrm{ZnO} / \mathrm{Ag} / \mathrm{ZnO}$ multilayers were fabricated and their optical properties were investigated in terms of the micro-cavity effect in electroluminescent devices based on colloidal quantum dots. The top and bottom ZnO layers were formed by a sol-gel method while the middle Ag layer was deposited by thermal evaporation. After the fabrication of the $\mathrm{ZnO} / \mathrm{Ag} / \mathrm{ZnO}$ structure, the transmittance increased to $74 \%$. When the oxide/metal/oxide multilayers were applied to quantum dot light-emitting diodes, the color purity was enhanced due to the narrower full width at half maximum.
\end{abstract}

Key words : ZnO, Optical properties, Sol-gel process

\section{Introduction}

$\mathrm{T}$ he micro-cavity effect is a key technology that will be deployed in next-generation displays due its enhancement of the color purity, as caused by a narrowing of the broad emission spectra of electroluminescent (EL) devices without complicated optics. ${ }^{1,2)}$ Generally, to attain an efficient micro-cavity effect in EL devices, a pair of reflective electrodes and a transparent electrode are required. Unlike the simple fabrication of a reflective electrode by metal deposition, many requirements must be satisfied to enable the use of transparent conducting oxides (TCOs) as a transparent electrode for application to flat-panel displays and touch screens. ${ }^{3,4)}$ Although indium tin oxide (ITO) thin films are the most commonly used TCO materials, the world's limited reserves of indium makes ITO more expensive., ${ }^{5,6)}$ $\mathrm{ZnO}$ has been investigated as a possible replacement for ITO electrodes as its deposition is relatively inexpensive and yet offers good optical/electrical properties. $\mathrm{ZnO}$ has good transparency in the visible range, with high electron mobility, and a wide bandgap of $3.37 \mathrm{eV}{ }^{7,8)}$ Recently, oxide/ metal/oxide (OMO) multilayers with very thin metal middle layer have exhibited enhanced electrical and optical properties resulting from the combination of the metal with a low refractive index and oxides with a high refractive index. ${ }^{9,10}$

In the present study, $\mathrm{ZnO} / \mathrm{Ag} / \mathrm{ZnO}$ multilayers were fabricated and applied to quantum dot light-emitting diodes (QLEDs), one of the most promising EL devices, ${ }^{11,12)}$ to verify the micro-cavity effect. Commonly, OMO multilayers are fabricated by the RF sputtering method, which is limited to large-area fabrication. In this case, however, OMO multi-

\footnotetext{
Corresponding author : Jiwan Kim

E-mail : jiwank@kgu.ac.kr

Tel : +82-31-249-1308 Fax : +82-31-249-9774
}

layers were fabricated using the convenient sol-gel method for the $\mathrm{ZnO}$ layers and thermal evaporation for the thin $\mathrm{Ag}$ layer. Their optical properties were systemically studied. When EL spectra from QLEDs with/without OMO multilayers were compared with each other, the narrowed full width at half maximum (FWHM) resulting from the micro-cavity effect of the OMO multilayers was observed.

\section{Experimental Procedure}

\subsection{Preparation of $\mathrm{ZnO} / \mathrm{Ag} / \mathrm{ZnO}$ multilayer}

The top and bottom $\mathrm{ZnO}$ layers, each $100 \mathrm{~nm}$ thick, were formed using a sol-gel method. First, zinc acetate dihydrate $\left(\mathrm{Zn}\left(\mathrm{CH}_{3} \mathrm{COO}\right)_{2} \cdot 2 \mathrm{H}_{2} \mathrm{O}\right)$ was dissolved in 2-methoxyethanol. The solution was then stirred for $2 \mathrm{~h}$ at $75^{\circ} \mathrm{C}$ and then the temperature was increased up to $200^{\circ} \mathrm{C}$ and remained for about $5 \mathrm{~min}$ to get clear solution of $\mathrm{ZnO}$. This clear solution was spin-coated onto the opposite side of an ITO patterned glass substrate at 5,000 rpm for $30 \mathrm{~s}$ and then placed on a hotplate at $50^{\circ} \mathrm{C}$ to evaporate the residual solvent. To form a dense, crystalized $\mathrm{ZnO}$ layer, the temperature of the hotplate was increased to $380^{\circ} \mathrm{C}$, and maintained at that temperature for $1 \mathrm{~h}$. The middle Ag layer $(6 \mathrm{~nm})$ was deposited by thermal evaporation at a pressure of $1.5 \times 10^{-6}$ torr.

\subsection{Fabrication of QLEDs}

First, the ITO glass, patterned to form an anode, was cleaned by sonication using isopropyl alcohol and then deionized water. The cleaned ITO anode was dried, after which an oxygen plasma treatment was applied to the surface of the ITO patterned glass for $5 \mathrm{~min}$. For the hole injection layer (HIL), poly(3,4-ethylenedioxythiophene) : poly (styrene sulfonate) (PEDOT : PSS) was spin-coated onto the ITO substrate at $3000 \mathrm{rpm}$ for $35 \mathrm{~s}$ and then dried on a hotplate at $180^{\circ} \mathrm{C}$ for $30 \mathrm{~min}$. For the hole transport layer 
(HTL), poly[(9,9-dioctylfluorene-co- $N$-[4-(3-methylpropyl)]diphenylamine] (TFB) was dissolved in chlorobenzene (8 $\mathrm{mg} / \mathrm{ml}$ ) and then spin-coated onto the PEDOT:PSS layer at $2,000 \mathrm{rpm}$ for $35 \mathrm{~s}$. It was then dried on a hotplate at $150^{\circ} \mathrm{C}$ for $30 \mathrm{~min}$. Similarly, for the EML, green CdSe/ZnS QDs were dispersed in heptane $(0.5 \mathrm{mg} / \mathrm{ml})$ and then spin-coated onto the TFB layer at 2,000 rpm for $5 \mathrm{~s}$. Likewise, for the ETL, ZnO NPs were synthesized using a protocol described in the literature. ${ }^{13)}$ The $\mathrm{ZnO}$ NPs were then re-dispersed in ethanol prior to spin-coating on the $\mathrm{QD}$ emission layer at $3,000 \mathrm{rpm}$ for $60 \mathrm{~s}$. After the completion of the solution process, the cathode ( $\mathrm{Al}$, about $120 \mathrm{~nm}$ ) was deposited by thermal evaporation on the ZnO NP layer. Finally, the devices were encapsulated in glass using a UV sealant.

\subsection{Characterization}

A PerkinElmer Lambda 19 spectrometer was used to measure the transmittance of the films over a wavelength range of $300-750 \mathrm{~nm}$. The electroluminescence (EL) spectra were measured by using a spectroradiometer (Minolta CS 2000) with a Keithley 2400 source meter under ambient conditions.

\section{Results and Discussion}

The transmittance spectra for a wavelength range of 350 $750 \mathrm{~nm}$ for $\mathrm{ZnO} / \mathrm{Ag} / \mathrm{ZnO}$ multilayers with $\mathrm{Ag}$ middle layers of different thicknesses are shown in Fig. 1. The OMO multilayers were fabricated using the convenient sol-gel method for the top and bottom $\mathrm{ZnO}$ layer and thermal evaporation for the middle Ag layer. It is well known that bare crystallized $\mathrm{ZnO}$ film has a visible spectrum transmittance of more than $80 \%{ }^{14)}$ Once the OMO multilayers have been formed, a degree of waviness can be observed over the entire visible region. In the OMO multilayers, the Ag layer acts as a mirror for the incident light, while the $\mathrm{ZnO}$ layers act as an anti-reflective coating. The light reflected by the front and rear surfaces of each $\mathrm{ZnO}$ layer are of opposite phase and of nearly equal amplitude, which results in a decrease in the

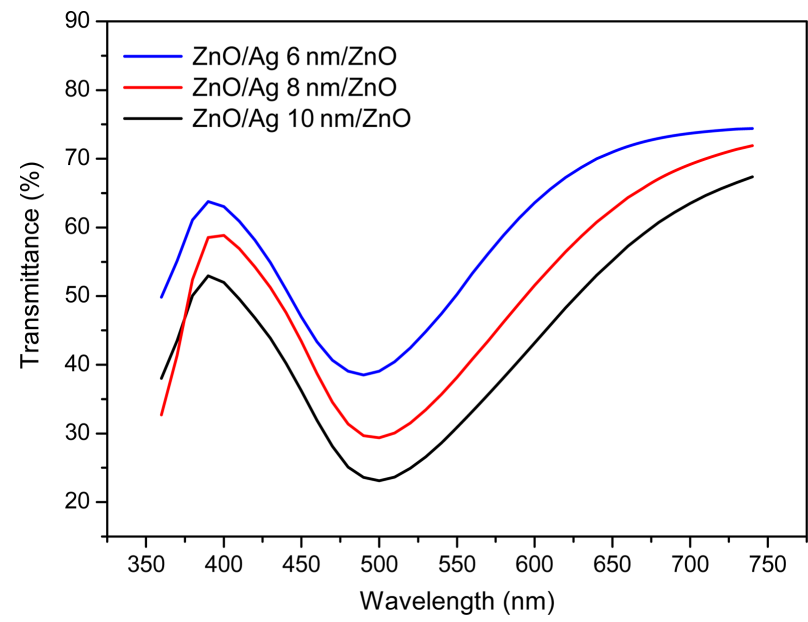

Fig. 1. Transmittance spectra of $\mathrm{ZnO} / \mathrm{Ag} / \mathrm{ZnO}$ multilayers. amount of light that is reflected. The region around $500 \mathrm{~nm}$ exhibits a relatively low value due to the excitation of the localized surface plasmons in the thin Ag layer. ${ }^{15)}$ This is caused by the micro-cavities formed in the OMO layers. Upon increasing the thickness of the middle Ag layer (from 6 to $10 \mathrm{~nm}$ ), the transmittance of the OMO multilayers decreases across most of the visible light spectrum. Interestingly, the transmittance of the OMO multilayers increases to $74 \%$ in the long wavelength region. It is assumed that a 6-nm Ag film is too thin to form a perfect layer, with some $\mathrm{Ag}$ atoms still existing as aggregated $\mathrm{Ag}$ islands, prior to forming a continuous layer. Therefore, longer-wavelength light (red and near-IR) can pass through it easily.

To investigate the spectral response after light passes through the OMO multilayers, QLEDs were fabricated and positioned $15 \mathrm{~mm}$ from the fabricated OMO multilayers. Fig. 2 is a schematic of the structure of a QLED device on an OMO multilayer. The QLEDs have the standard structure with ITO/PEDOT : PSS/TFB/green QDs/ZnO NPs/Al. The QLEDs were carefully designed to achieve efficient carrier injection and balance in the QD emission layer. In this device structure, PEDOT : PSS was used as the HIL on the ITO anode to increase the anode work function and compensate for the surface roughness, thus producing a stable interface. TFB was used as the HTL to reduce the energy barrier between the work function of the PEDOT : PSS and the valence-band edge of the green QDs. Due to the chemical stability of the TFB thin films to nonpolar solvents, QDs can be simply spin-coated over the TFB layer. $\mathrm{ZnO}$ NPs have become well known as inorganic materials for application to ETL in recent QLEDs due to their robustness and efficient electron injection. ${ }^{13,16)}$

Figure 3 shows the current efficiency as a function of the luminance for the fabricated QLEDs. The luminance increases with the voltage, attaining a maximum of 5,700 $\mathrm{cd} / \mathrm{m}^{2}$ under an applied voltage of $8 \mathrm{~V}$. A maximum current efficiency of $3.6 \mathrm{~cd} / \mathrm{A}$ was achieved at $200 \mathrm{~cd} / \mathrm{m}^{2}$. Since the OMO multilayers separate from the QLEDs, they have no effect on the performance of the QLEDs.

The changes in the EL spectrum of the QLEDs with $\mathrm{ZnO} /$ $\mathrm{Ag} / \mathrm{ZnO}$ multilayers are shown in Fig. 4(a). For the bare

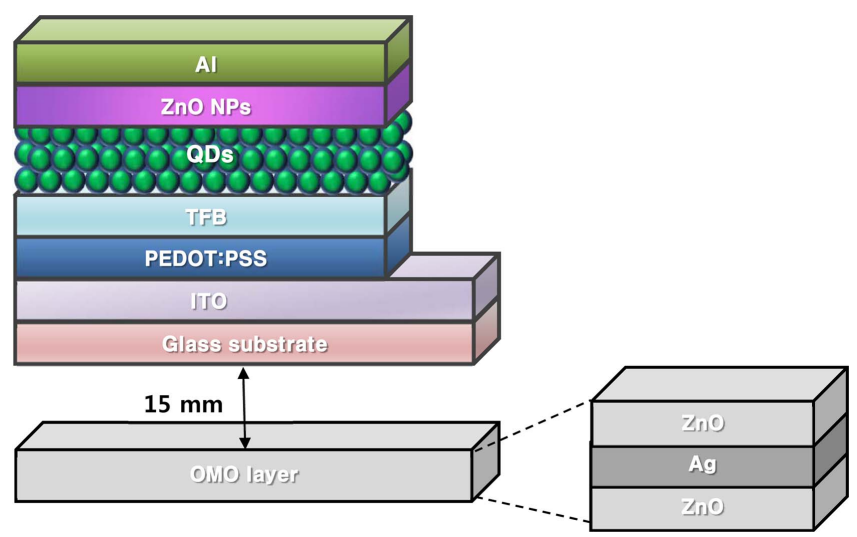

Fig. 2. Schematic of QLEDs on OMO multilayers. 


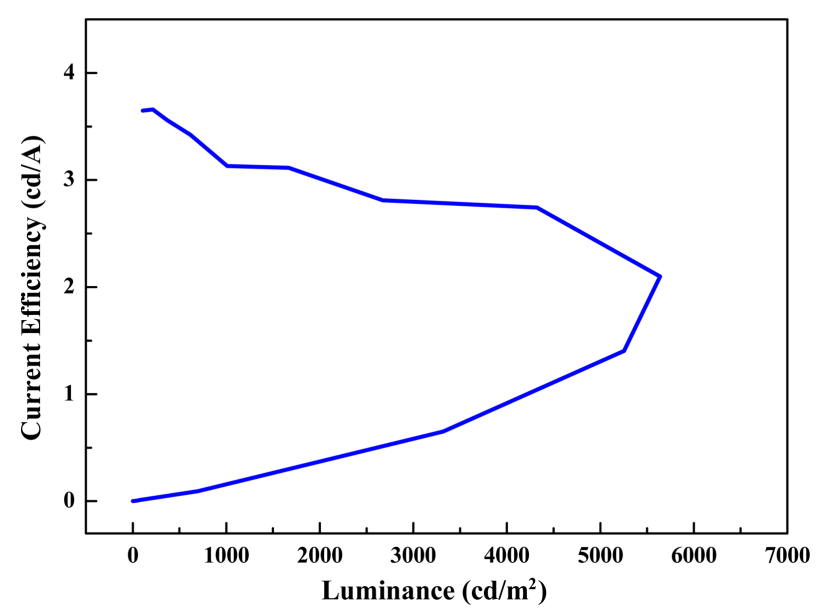

Fig. 3. Current efficiency as function of luminance of QLEDs on OMO multilayers.

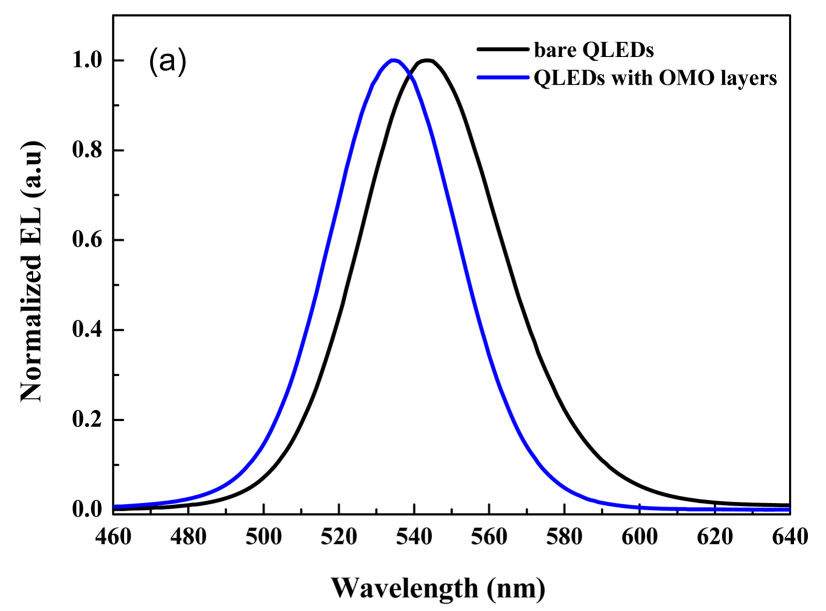

(b)

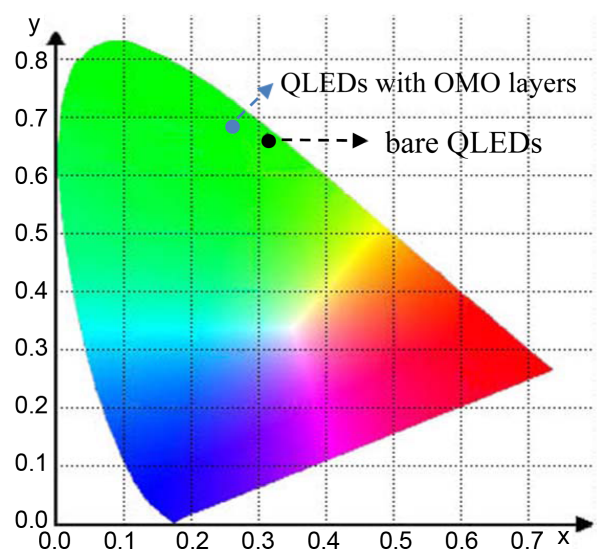

Fig. 4. (a) Change in EL spectrum and (b) CIE 1931 color diagram showing color coordinates of green QLEDs with OMO multilayers.

QLEDs, a narrow peak with a FWHM value of $45 \mathrm{~nm}$ is observed at $540 \mathrm{~nm}$ without any notable parasitic emission from the charge-transport layers. When the OMO multilayers are applied to the QLEDs, the normalized EL spectrum is blue-shifted by $9 \mathrm{~nm}$ and the FWHM becomes narrower, being only $40 \mathrm{~nm}$. As shown in Fig. 1, the green spectral emission (near $500 \mathrm{~nm}$ ) is limited by the low transmittance due to the micro-cavity effect. Relative to a device without any OMO multilayers, the FWHM decreased by $5 \mathrm{~nm}$. The change in the green color is visualized by plotting the color coordinates on a CIE 1931 standard color diagram, as shown in Fig. 4(b). Generally, the color purity is sensitive to the FWHM of the emission peak and the plotted dots at the edge of the diagram represent the pure color. ${ }^{17)}$ By optimizing the OMO multilayers, a relatively narrow EL spectrum is obtained from the QLEDs. This enhances the color purity.

\section{Conclusions}

We investigated the optical properties of $\mathrm{ZnO} / \mathrm{Ag} / \mathrm{ZnO}$ multilayers. $\mathrm{ZnO}$ layers were formed using the convenient sol-gel method while the mid-metal layers were formed using thermal evaporation. After the fabrication of the OMO structure, the transmittance increased to $74 \%$. When the OMO multilayers were applied to QLEDs, the normalized EL peak was narrowed due to the micro-cavity effect. These results show that OMO multilayers can be used to enhance the color purity of future display applications.

\section{Acknowledgments}

This work was supported by the Basic Science Research Program through the National Research Foundation of Korea (NRF), funded by the Ministry of Science and ICT (2017R1A2B4012274). This work was also supported by the Korea Institute of Energy Technology Evaluation and Planning (KETEP) and the Ministry of Trade, Industry \& Energy (MOTIE) of the Republic of Korea (No. 20163030013980).

\section{REFERENCES}

1. P. E. Burrows, G. Gu, S. R. Forrest, E. P. Vicenzi, and T. X. Zhou, "Semitransparent Cathodes for Organic Light Emitting Devices," J. Appl. Phys., 87 [6] 3080-85 (2000).

2. R. B. Pode, C. J. Lee, D. G. Moon, and J. I. Han, "Transparent Conducting Metal Electrode for Top Emission Organic Light-Emitting Devices: Ca-Ag Double Layer," Appl. Phys. Lett., 84 [23] 4614-16 (2004).

3. M. Purica, E. Budianu, E. Rusu, M. Danila, and R. Gavrila, "Optical and Structural Investigation of ZnO Thin Films Prepared by Chemical Vapor Deposition (CVD)," Thin Solid Films, 403-404 485-88 (2002).

4. M. H. Ahn, E. S. Cho, and S. J. Kwon, "Characteristics of ITO-Resistive Touch Film Deposited on a PET Substrate by In-Line DC Magnetron Sputtering," Vacuum, 101 22127 (2007).

5. T. Minami, H. Sonohara, T. Kakumu, and S. Takata, "Physics of Very Thin ITO Conducting Films with High Transparency Prepared by DC Magnetron Sputtering," Thin Solid Films, 270 37-42 (1995).

6. F. Ruffino, E. Carria, S. Kimiagar, I. Crupi, F. Simone, and M. G. Grimaldi, "Formation and Evolution of Nanoscale Metal Structure on ITO Surface by Nanosecond Laser 
Irradiation of Thin Au and Ag Films," Sci. Adv. Mater., 4 [7] 708-18 (2012).

7. V. Bhosle, A. Tiwari, and J. Narayan, "Metallic Conductivity and Metal-Semiconductor Transition in Ga-Doped ZnO," Appl. Phys. Lett., 88 [3] 032106 (2006).

8. E.-S. Jang, "Recent Progress in Synthesis of Plate-like $\mathrm{ZnO}$ and its Applications: A Review," J. Korean Ceram. Soc., 54 [3] 167-83 (2017).

9. H. Han, N. D. Theodore, and T. L. Alford, "Improved Conductivity and Mechanism of Carrier Transport in Zinc Oxide with Embedded Silver Layer," J. Appl. Phys., 103 [1] 013708 (2008).

10. D. R. Sahu, and J. L. Huang, "High Quality Transparent Conductive $\mathrm{ZnO} / \mathrm{Ag} / \mathrm{AnO}$ Multilayer Films Deposited at Room Temperature," Thin Solid Films, 515 876-79 (2006).

11. T. H. Kim, K. S. Cho, E. K. Lee, S. J. Lee, J. Chae, J. W. Kim, D. H. Kim, J. Y. Kwon, G. Amaratunga, S. Y. Lee, B. L. Choi, Y. Kuk, J. M. Kim, and K. Kim, "Full-Colour Quantum Dot Displays Fabricated by Transfer Printing," Nat. Photonics, 5 176-82 (2011).

12. C. Y. Han, and H. Yang, "Development of Colloidal Quantum Dots for Electrically Driven Light-Emitting Devices," J. Korean Ceram. Soc., 54 [6] 449-69 (2017).
13. K. H. Lee, J. H. Lee, H. D. Kang, B. Park, Y. Kwon, H. Ko, C. Lee, J. Lee, and H. Yang, "Over $40 \mathrm{~cd} / \mathrm{A}$ Efficient Green Quantum Dot Electroluminescent Device Comprising Uniquely Large-Sized Quantum Dots," ACS Nano, 8 [5] 4893-901 (2014).

14. Z. Lin, J. Chang, C. Jiang, J. Zhang, J. Wu, and C. Zhu, "Enhanced Inverted Organic Solar Cell Performance by Post-Treatments of Solution-Processed ZnO Buffer Layers," RSC Adv., 4 [13] 6646-51 (2014).

15. T. L. Temple, G. D. K. Mahanama, H. S. Reehal, and D. M. Bagnall, "Influence of Localized Surface Plasmon Excitation in Silver Nanoparticles on the Performance of Silicon Solar Cells," Sol. Energy Mater. Sol. Cells, 93 [11] 1978-82 (2009).

16. L. Qian, Y. Zheng, J. Xue, and P. H. Holloway, "Stable and Efficient Quantum-Dot Light-Emitting Diodes Based on Solution-Processed Multilayer Structures," Nat. Photonics, 5 543-48 (2011).

17. J. Lim, W. K. Bae, J. Kwak, S. Lee, C. Lee, and K. Char, "Perspective on Synthesis, Device Structures, and Printing Processes for Quantum Dot Displays," Opt. Mater. Express, 2 [5] 594-628 (2012). 